\title{
Evaporation-Field Differences with Deep-UV Atom Probe Tomography
}

Ty Prosa ${ }^{1}$, Dan Lenz ${ }^{2}$, Isabelle Martin ${ }^{2}$, David Reinhard ${ }^{3}$, David Larson ${ }^{3}$ and Joe Bunton ${ }^{2}$

${ }^{1}$ CAMECA® Instruments Inc., 5470 Nobel Drive, Madison, WI 53711 USA, Wisconsin, United States, ${ }^{2}$ CAMECA® Instruments Inc., 5470 Nobel Drive, Madison, WI 53711 USA, United States, ${ }^{3}$ CAMECA Instruments Inc., Madison, Wisconsin, United States

Pulsed-laser atom probe tomography (PLAP) has been shown to be a thermally-activated fieldevaporation process [1]. The first PLAP investigations were performed in the 1970s and utilized $7 \mathrm{~ns}$, $337 \mathrm{~nm}$ laser pulses [2]. Since the 2000s, commercially available instruments have seen a steady progression from longer wavelengths into the UV: The LAWATAP (CAMECA) eventually could switch between $1030 \mathrm{~nm}, 515 \mathrm{~nm}$ and $343 \mathrm{~nm}$ (with a prototype using $778 \mathrm{~nm}$ [3]), the LEAP 3000 (Imago) initially provided $535 \mathrm{~nm}$ and progressed to UV $355 \mathrm{~nm}$ for the CAMECA LEAP 4000 and 5000 (including a $400 \mathrm{~nm}$ prototype instrument) [4]. Recent investigations with extreme-UV suggest that new physics may come into play with photons capable of directly ionizing inner shell electrons (29.6 $\mathrm{nm}$ ) [5]. Although there have been reports that some materials might benefit from analysis with longer wavelengths, the consensus has become that shorter wavelengths provide benefits for data quality, specimen-laser interaction, and yield [4,6,7]. In this presentation, we will discuss some preliminary results using a deep-UV (266 nm) laser system to look for novel benefits.

During the course of this investigation, multiple materials have been analyzed with deep-UV. The most dramatic effect was noted on silicon/silicon-dioxide materials and interfaces. Differences in evaporation field are known to limit specimen survivability and reconstruction accuracy because of non-uniform evaporation [8]. Fig. 1 shows comparisons between $266 \mathrm{~nm}$ and $355 \mathrm{~nm}$ laser-pulsed APT acquisitions of similarly sized specimens made from our internal standard $\left(\mathrm{Si} / \mathrm{SiO}_{2}-12-\mathrm{nm} / \mathrm{Si}\right)$ [8]. The voltage trends suggest that the relative evaporation field differences between $\mathrm{Si}$ and $\mathrm{SiO}_{2}$ are dramatically reduced for $266 \mathrm{~nm}$ relative to $355 \mathrm{~nm}$ (Fig. 1b), and leads to much more uniformity in evaporation, especially for the transition from $\mathrm{SiO}_{2}$ into $\mathrm{Si}$ (Fig. 1c vs. 1d). Ultimately, the smoother transition between layers leads to better, more homogeneous reconstructions (Fig. 1e vs. 1f) and suggests higher survivability as well. Preliminary data shows higher survivability under a greater variety of analysis conditions for this material. We note that the $266 \mathrm{~nm}$ data was collected with significantly higher average applied field and detection rates (DR) than reported in [8]. The $266 \mathrm{~nm}$ analysis was collected at 10\% DR and average silicon charge-state-ratio (CSR) near 100, while $355 \mathrm{~nm}$ analysis was collected under modest $0.5 \%$ DR and 20 CSR conditions. 

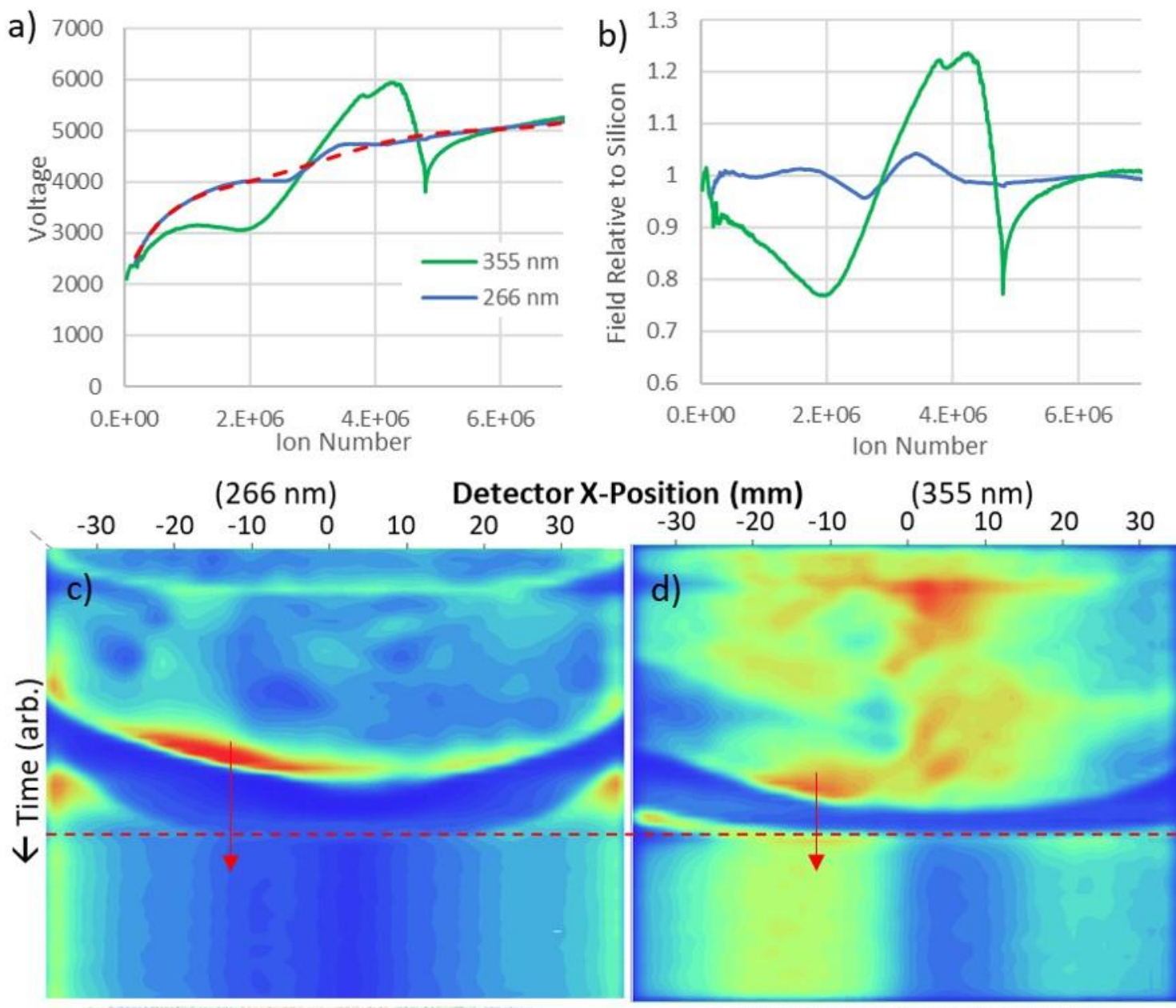

e)
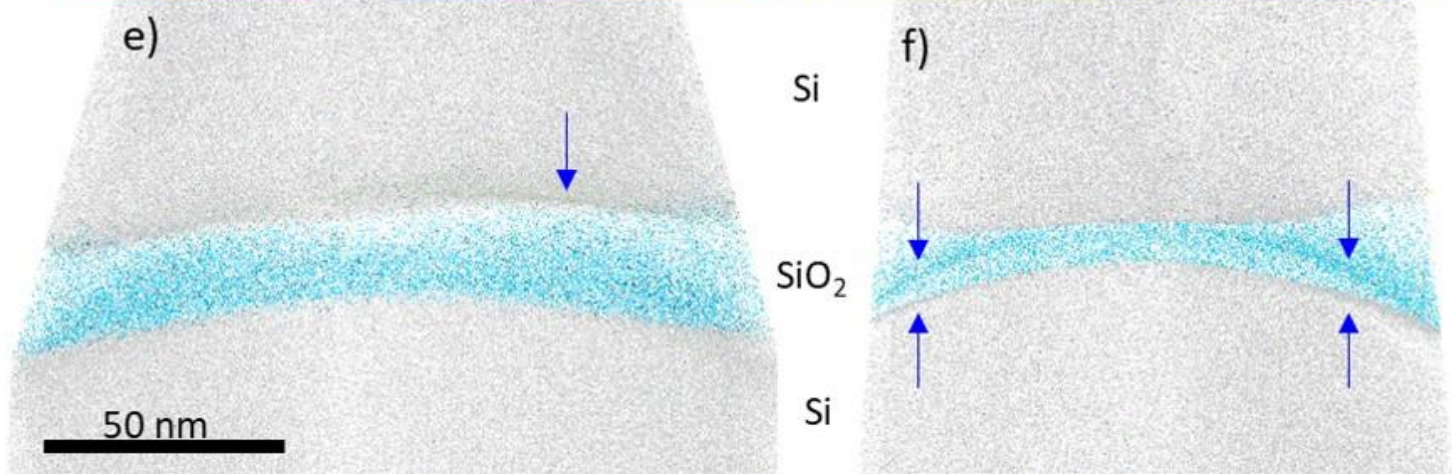

Figure 1. Figure 1. $266 \mathrm{~nm}$ and $355 \mathrm{~nm}$ PLAP comparison of a) acquisition voltage, b) relative evaporation field, c) and d) detection uniformity, e) and f) reconstruction uniformity for a standard 12nm-oxide layer surrounded by $\mathrm{Si}$ [8]. a) Voltage trends are compared with an estimated average voltage trend for pure $\mathrm{Si}$ (red-dashed line in a) and converted into b) evaporation field relative to Si. 10-mm-thick hit-density maps (arbitrary color scale - red high density, blue low) are shown in c) and d). The reddashed-line represents the detected ion-density at a single moment in time across the center of the detector; This moment captures the transition from $\mathrm{SiO} 2$ back into $\mathrm{Si}$, with the high uniformity in c) resulting in more accurate reconstruction for e). Red arrows indicate regions for comparison. e) and f) show high reconstruction density aberrations (blue arrows) that have resulted from non-uniform evaporation. 


\section{References}

[1] F. Vurpillot et al., J. Phys. D: Appl. Phys. 42 (2009) 125502.

[2] G. L. Kellogg and T. T. Tsong, Journal of Applied Physics 51 (1980) 1184.

[3] B. Gault et al., Review of Scientific Instruments 77 (2006) 043705/1.

[4] J. H. Bunton et al., Microscopy and Microanalysis 13 (2007) 418.

[5] A. N. Chiaramonti et al., Microscopy and Microanalysis 26 (2020) 258.

[6] K. Hono et al., Ultramicroscopy 111 (2011) 576.

[7] A. Vella et al., Journal of Applied Physics 110 (2011) 044321/1.

[8] T. J. Prosa et al., Microscopy and Microanalysis 21 (2015) 849. 